\title{
Efficacy of Cognitive-Behavioral Group Therapy Versus Group Hypnotherapy on Brain/Behavioral Systems of Social Phobia Patients
}

\author{
Golnaz Forouzandehfar $^{1}$ (D), Mehdi Fathi ${ }^{*}$ (D), Mohsen Dadashi ${ }^{3,4} *(D)$ \\ 1. Dept. of Clinical Psychology, Faculty of Psychology, Shiraz University, Shiraz, Iran \\ 2. Surgical Oncology Research Center, Faculty of Medicine, Mashhad University of Medical Sciences, Mashhad, Iran \\ 3. Dept. of Clinical Psychology, Faculty of Medicine, Zanjan University of Medical Sciences, Zanjan, Iran \\ 4. Social Determinants of Health Research Center, Zanjan University of Medical Sciences, Zanjan, Iran
}

\begin{tabular}{|c|c|}
\hline Article Info & ABSTRACT \\
\hline doi $10.30699 /$ jambs.27.122.51 & Background \& Objective: Social anxiety disorder, which involves excessive \\
\hline $\begin{array}{l}\text { Received: 2019/01/10; } \\
\text { Accepted: 2019/03/15; } \\
\text { Published Online: } 10 \text { May 2019; }\end{array}$ & $\begin{array}{l}\text { that leads to social dysfunction. The purpose of this study is to investigate Efficacy } \\
\text { of Cognitive-Behavioral Group Therapy (CBGT) versus Group Hypnotherapy (GHt) } \\
\text { on Brain/Behavioral Systems of Social Phobia Patients. }\end{array}$ \\
\hline $\begin{array}{l}\text { Use your device to scan and read the } \\
\text { article online }\end{array}$ & $\begin{array}{l}\text { Materials \& Methods: The numbers of } 30 \text { subjects were divided into two groups } \\
\text { receiving either CBGT or GHt. The questionnaires of brain/behavioral systems } \\
\text { (GWPQ) and social phobia (SPIN) were administered in two phases (before and after } \\
\text { intervention). }\end{array}$ \\
\hline Ond & $\begin{array}{l}\text { Results: Both therapy approaches caused significant changes in the level of social } \\
\text { phobia and brain/behavioral systems activity. The alterations in brain-behavioral } \\
\text { subsystems (BIS and FFFS) in the Extinction and Flight components were in favor } \\
\text { of hypnotherapy }(P<0.05) \text {. }\end{array}$ \\
\hline (1) 30. & $\begin{array}{l}\text { Conclusion: The findings from the present research provide evidence that due to } \\
\text { alterations in brain/behavioral systems associated with the social phobia, }\end{array}$ \\
\hline $\begin{array}{l}\text { Corresponding Information: } \\
\text { Dadashi M, Dept. of Clinical Psychology, } \\
\text { Faculty of Medicine, Zanjan University of } \\
\text { Medical Sciences, Zanjan, Iran } \\
\text { Email: Psy.mohsen@gmail.com }\end{array}$ & $\begin{array}{c}\text { Keywords: Social phobia, Brain/behavioral systems, BIS, FFFS, Cognitive- } \\
\text { behavioral therapy, Hypnotherapy }\end{array}$ \\
\hline (c) (1) (8) $\quad \begin{array}{l}\text { Copyright } \odot 2019 \text {, This is a } \\
\text { copy and redistribution of } \mathrm{tl}\end{array}$ & $\begin{array}{l}\text { er the terms of the Creative Commons Attribution-noncommercial } 4.0 \text { International License which permits } \\
\text { th proper citation. }\end{array}$ \\
\hline
\end{tabular}

\section{Introduction}

The social anxiety is recognized to be one of the most common malfunctions among both anxiety related disorders and mental disorders (1).

As it is defined in DSM 5, Social Anxiety (which is also referred to as Social Phobia) constitutes a consistent fear in regard to diverse situational contexts and their relevant performances which can lead to embarrassment or humiliation of the individual. Although the individuals acknowledge that the fear is unreasonable or excessive, yet exposure to the feared situation can cause physiological symptoms, avoidance and as a result a major dysfunction in social and occupational activities (2).

The prevalence estimation indicates that approximately $7-13 \%$ of the Western societies' population meets diagnostic criteria for the social phobia at some point during their lifetime (3).

There are various pathways associated with the acquisition and maintenance of SAD such as genetic and temperamental influences, environmental factors (e.g. parental factors, peer influences, conditioning or learning experiences) and cognitive styles and biases (46). The behavioral inhibition which is affected by temperament and personality is significantly associated with social anxiety. The behavioral inhibition is a tendency which is marked by the restriction of behavior, a low level of approach-oriented acts, enhanced vigilancy, and intensified reaction to strange conditions (7). Gary's theory of personality treats the inhibition of behavior as one of the main aspects of personality and refers to it as the reinforcement sensitivity theory (RST) $(8,9)$. RST suggest three major subsystems in brain which underlies many of the personality's individual differences; psychopathology, reinforcement sensitivity and motivated behavior $(\mathbf{1 0}, \mathbf{1 1})$. The aforementioned systems in brain constitute the Fight-Flight-Freeze System (FFFS), Behavioral Approach System (BAS), and Behavioral Inhibition System (BIS).

\section{Brain/Behavioral Systems}

\section{Behavioral Approach System (BAS)}

The BAS represents a subsystem which is related to the appetite-oriented motivation in brain. This subsystem reacts to the conditioned and unconditioned stimuli by means of approach-related responses. (10). The BAS neural structure is the mesolimbic dopamine pathway, 
rising from the ventral segmental part of brain to the striatum and the nucleus accumbens (11). Therefore, hypoactivity in the BAS has been assumed to cause depression whereas, hyperactivity has been considered to mediate mania (11).

\section{Behavioral Inhibition System (BIS)}

The subsystem which controls defensive behavior in brain is the BIS. More specifically, it focuses on resolving approach-avoidance conflict while facing competing goals by increasing attention, emotional arousal, restriction of prepotent responses, and triggering risk assessment (12). These risk assessment behaviors can be described as environmental scanning and memory scanning for the threat-relevant information (10). It seems that the BIS has an avoidance approach towards threatening stimuli (13). The BIS has claimed to be the neural network related to anxiety, since the major components of the BIS comprise medial hypothalamus, septo-hippocampal system, the periaqueductal gray, amygdala, prefrontal dorsal stream, and posterior cingulate (12), The hyperactivity in BIS can lead to several mental disorders including the generalized anxiety and depression $(11,13)$.

\section{Fight-Flight-Freeze System (FFFS)}

The FFFS prompts the restrain of all aversive stimuli (10). Therefore, it has been known as the brain subsystem motivating scape and avoidance responses. Since the neural structure of the FFFS comprise the periaqueductal gray, amygdala, medial hypothalamus, prefrontal ventral stream, and anterior cingulate (12), it is supposed to be engaged with the emotions of fear and panic. Hyperactivity in the FFFS can demonstrate several anxiety disorders, including panic disorder and specific phobia (13).

Gray and McNaughton (2000) and Kimbrel (2008) argued that increase in BIS sensitivity is the neurobiological mechanism underlie Social Anxiety Disorder. A large number of studies endorsed this proposition (e.g., Coplan et al., 2006; Kashdan \& Roberts, 2006; Kimbrel et al., 2008). Furthermore, a number of imaging studies (e.g., Tillfors et al., 2001; Tillfors et al., 2002) have shown that individuals with social anxiety demonstrate rises in regional cerebral blood flow in parts of the BIS $(4,13-17)$. Further studies indicate that the sensitivity of fight-flight-freeze system (FFFS) had a positive relationship with social observation anxiety and social interaction anxiety (18). In other word, heightened FFFS is a key motivational factor underlying social anxiety as well as BIS (4).

There are several well-established treatments for the anxiety disorders such as behavioral therapy, cognitivebehavioral therapy, hypnosis and Pharmacological treatment (19-21). Exposure to feared social stimuli, such as graduated exposure, flooding, and systematic desensitization, are claimed to decrease avoidance and symptoms of anxiety as a result of habituation to social stimuli occurring in the BIS and FFFS circuits for treatment of Social Anxiety. Researches suggest that
CBT appears to reduce social anxiety symptoms by decreasing sensitivity in BIS and FFFS (13). On the other hand, recent conducted research by Halsband $e t$ $a l$., showed that hypnosis can affect the left amygdala and bilaterally in the anterior cingulate cortex (ACC), insula and hippocampus by significant activation reduction of these areas, indicating that hypnosis can inhibit the reaction of the fear circuitry structures (22).

The purpose of this study was to compare the efficacy of short-term interventions in treating social phobia through different psychometric measures. Also, it is important to determine whether the changes in the brainbehavioral systems after treatment, differs from one therapy method to another or not.

\section{Materials and Methods}

\section{Participants}

The present study involved social anxiety patients, who participated in either CBGT or Hypnotherapy for the anxiety disorders at "Beheshti Psychiatry Hospital" during February 2016-August 2016. While 52 patients volunteered for the program, following diagnostic interview and further assessments only 37 entered the groups for treatment in the course of the study (i.e. two groups). Among these participants 34 individuals finished the programs. Divided into two Groups, first group received 6 sessions of CBGT whereas the second group received 4 sessions of Group Hypnotherapy. Due incomplete data, four of the participants completing the program were excluded from the study. The Participants were predominantly female (female $=17$, male $=13$ ), age 18-36 (CBGT $\mathrm{M}=26.2, \mathrm{SD}=4.93)(\mathrm{GHt} \mathrm{M}=24.8$, $\mathrm{SD}=3.41)$. Treatment completers in both groups did not differ by age, education, sex, ethnic background, number of diagnoses of anxiety related disorders, or comorbid depression.

\section{Procedures}

Patients were initially recruited to the study through posters and flyers in universities in Zanjan and also referred to the "Beheshti Psychiatry Hospital" from primary care clinic for the treatment of social anxiety disorder. Diagnostic interview was administered in order to determine the presence of social anxiety criteria. The individuals with SAD were randomly divided into two experimental groups (CBGT and GHt). While the First group received 6 therapy sessions of CBGT, the second group received 4 therapy sessions of hypnotherapy. The CBGT protocol was proposed by Morris, Mensink, and Sherry (Dalhousie University) driven largely from the treatment program developed by Heimberg (1991) and Heimberg and Becker (2002) and the GHt protocol was instructed by the researcher with approval license and supervision through the study course from the Iranian Scientific Society of Clinical Hypnosis. The participants were assessed in two stages of pre-test and post-test by following the questionnaires: social anxiety questionnaire and Gary Wilson's personality 
questionnaire. All the study procedures were approved by IRCT (Iranian Registry of clinical trials, IRCT Code: IRCT2015121425523N1), Zanjan University of Medical Sciences (ZUMS Code: IR.ZUMS.REC.1395.36), ISSCH (Iranian Scientific Society of Clinical Hypnosis) and Human Research Protection Program.

\section{Summary of the Cognitive-Behavior Group} Therapy (CBGT) Sessions

Each week the CBGT group had 2-h sessions. Two therapists were assigned to co-led therapy sessions for groups that each included 7 to 9 participants. The main therapeutic components of CBGT involved psychoeducation, cognitive restructuring, exposure to anxiety provoking stimuli, breathing exercises, and social skill training.

\section{Session 1}

1. Administering questionnaires

2. Introduction

3. Sharing common symptoms and similar feared situations among group members

4. Overview of the coming sessions

5. Determining feared situations

6. Discussion of thoughts/situations on the board

7. Explaining how thoughts can affect the subsequent physiological responses and behaviors

8. Introduction of the Vertical Arrow Technique

9. Assigning homework

\section{Session 2}

1. Review homework

2. Introduction of the 10 Burns cognitive distortions

3. Administration of Feared Situations Questionnaire

4. Assigning homework

\section{Session 3}

1. Review homework

2. Create automatic thoughts and responses on the board

3. Introducing the safety-behaviors/cognitions with examples

4. Homework

\section{Session 4}

1. Review homework

2. Introducing aspects of self-esteem

\section{Sessions 5 and 6}

1. Administering feared scenarios based on the responses from the Feared Situations Questionnaire.
2. Rating SUD (The Subjective Units of Distress) prior to commencing the role play, during and afterwards

3. Sharing feedbacks

4. Assigning exposure homework

5. Answering participants' questions and solving their problems in exposure tasks

Summary of the Group Hypnotherapy (GHt) sessions

\section{Session 1}

1. Administration of PMR (Progressive Muscle Relaxation) and relaxation training

2. Homework: records of the session were given to participants for further practice as homework

\section{Session 2}

1. Recognition of primary symptoms

2. Imagery inductions

3. Homework: records of the sessions 1 and 2 will be given to the participants for further practice

Session 3

1. Emotional modeling

2. Cognitive reconstruction

3. Behavior modification through mental visualization

4. Conditioning

5. Homework: records of the sessions 1,2 and 3 will be given to the participants for further practice

\section{Session 4}

1. Repetition of the conditioning

2. Repetition of the 3 rd session

3. Answering the participants' questions

4. Asking them for feedback

\section{Measures}

ADIS-IV Diagnostic interview (anxiety disorder interview schedule for DSM-IV):

Anxiety disorder interview for DSM-IV constitutes a structured interview for evaluating the present courses of anxiety disorders. The interview is based on DSM-IV's criteria for social anxiety. ADIS-IV facilitates differential diagnosis among anxiety disorders. Moreover, ADIS-IV furnishes adequate information for the pragmatic analysis of anxiety disorders (19).

\section{Social phobia Inventory (SPIN)}

SPIN was first proposed by Connor et al. Based on the results of the clinical uses of this questionnaire, it is helpful in the determination of the symptoms of phobia, avoidance, and physiological features. It has satisfactory reliability and validity indices. The reliability of this questionnaire was assessed by means of retesting (the scores were 0.89 and 0.87 ) in groups suffering from 
social phobia. Its internal consistency coefficient (Ella factor) was 0.94 for normal individuals. Moreover, for fear, avoidance, and physiological discomfort, this coefficient was $0.89,0.91$, and 0.80 respectively. In regard to Iranian samples including the groups who had social phobia disorder, the reliability was assessed by means of retesting and ranged from 0.78 to 0.98 . The alpha coefficient of this questionnaire was 0.96 in a group of normal individuals for the complete scale. Moreover, for the phobia, avoidance, and physiological discomfort subscales this coefficient was $0.89,0.91$, and 0.80 respectively $(20)$.

\section{Gary-Wilson personality questionnaire (GWPQ)}

This questionnaire assesses the level of activity of neurobehavioral systems along with their constituents. Wilson et al. (1989) developed GWPQ which comprises a self-assessment personality questionnaire. This questionnaire comprises 120 items. Forty items assess each one of the three systems including behavior activation system (BAS), behavior inhibition system (BIS), and fight-flight freeze systems (FFFS). Regarding BAS, 20 items evaluate the turned constituent and 20 items deal with the active avoidance constituent. In regard to BIS, 20 items focus on the potential active avoidance constituent and 20 items evaluate the silence constituent. Lastly, regarding the FFFS, 20 items examine the fight constituent and 20 items determine the flight constituent. Wilson et al., (1989) noted that the alpha coefficients ranged mostly from 0.6 to 0.7 and showed the satisfactory internal consistency of the questionnaire. As Azad Fallah et al. (2001) stated, the Cronbach's alpha coefficients for the turned constituent, active avoidance constituent, potentially active avoidance constituent, silence constituent, fight constituent, and flight constituent were 0.68, 0.65, $0.78,0.71,0.69$, and 0.78 respectively $(23)$.

\section{Results}

Seven subjects were dropped out of the experiment due to the absence from therapy sessions and incomplete data. For data analysis, SPSS 21 (SPSS Inc. Chicago, Illinois, USA) was used. Kolomogrov-Smirnov and Levene tests were used for determining normal distribution of the data and the variance homogeneity, respectively. Since pre-assumption of variance homogeneity and normal distribution had been observed, the parametric tests were used. The results showed that there was no difference between experimental groups in terms of research variables, age and educational variables in pretest. The ANCOVA results related to the research variables are presented in Table 1.

Table 1. Mean, Standard Deviation and post treatment ANCOVA results

\begin{tabular}{|c|c|c|c|c|c|c|c|c|c|c|c|}
\hline \multirow{3}{*}{ Variables } & \multicolumn{4}{|c|}{ CBGT } & \multicolumn{4}{|c|}{$\mathrm{GHt}$} & \multirow{3}{*}{$\underline{F}$} & \multirow{3}{*}{$\underline{P}$} & \multirow{3}{*}{$\frac{\text { Partial Eta }}{\underline{\text { Squared }}}$} \\
\hline & \multicolumn{2}{|c|}{ Pretest } & \multicolumn{2}{|c|}{ Posttest } & \multicolumn{2}{|c|}{ Pretest } & \multicolumn{2}{|c|}{ Posttest } & & & \\
\hline & $\underline{\mathrm{M}}$ & $\underline{\mathrm{SD}}$ & $\underline{\mathrm{M}}$ & $\underline{\mathrm{SD}}$ & $\underline{\mathrm{M}}$ & $\underline{\mathrm{SD}}$ & $\underline{\mathrm{M}}$ & $\underline{\mathrm{SD}}$ & & & \\
\hline SPIN & 37.07 & 7.98 & 9.27 & 2.93 & 37.20 & 5.90 & 9.33 & 2.87 & 0.002 & 0.966 & 0.000 \\
\hline Approach & 17.20 & 3.84 & 14.93 & 4.95 & 16.80 & 4.20 & 14.27 & 3.45 & 0.13 & 0.722 & 0.005 \\
\hline $\begin{array}{c}\text { Active } \\
\text { Avoidance }\end{array}$ & 29.60 & 2.85 & 26 & 3.70 & 29.07 & 2.12 & 26 & 2.83 & 0.014 & 0.905 & 0.001 \\
\hline $\begin{array}{l}\text { Passive } \\
\text { Avoidance }\end{array}$ & 13.33 & 2.69 & 8.53 & 4.50 & 13.60 & 2.52 & 8.13 & 3.16 & 0.156 & 0.696 & 0.006 \\
\hline Extinction & 23.20 & 3.84 & 13.33 & 2.58 & 23.47 & 3.96 & 11.20 & 3.69 & 6.963 & $0.014 *$ & 0.205 \\
\hline Fight & 11.60 & 2.41 & 16 & 3.12 & 12.27 & 2.12 & 19.07 & 5.34 & 2.871 & 0.102 & 0.096 \\
\hline Flight & 24.40 & 6.15 & 20.67 & 4.58 & 23.20 & 6.13 & 17.47 & 3.50 & 5.957 & $0.021 *$ & 0.181 \\
\hline BAS & 46.80 & 4.06 & 40.93 & 5.28 & 45.87 & 5.15 & 40.27 & 3.92 & 0.000 & 0.988 & 0.000 \\
\hline BIS & 36.53 & 5.42 & 21.87 & 6.12 & 36.93 & 4.89 & 19.33 & 5.33 & 2.419 & 0.132 & 0.082 \\
\hline FFFS & 36.53 & 7.61 & 36.67 & 5.89 & 36.40 & 7.02 & 36.53 & 5.73 & 0.002 & 0.968 & 0.000 \\
\hline
\end{tabular}




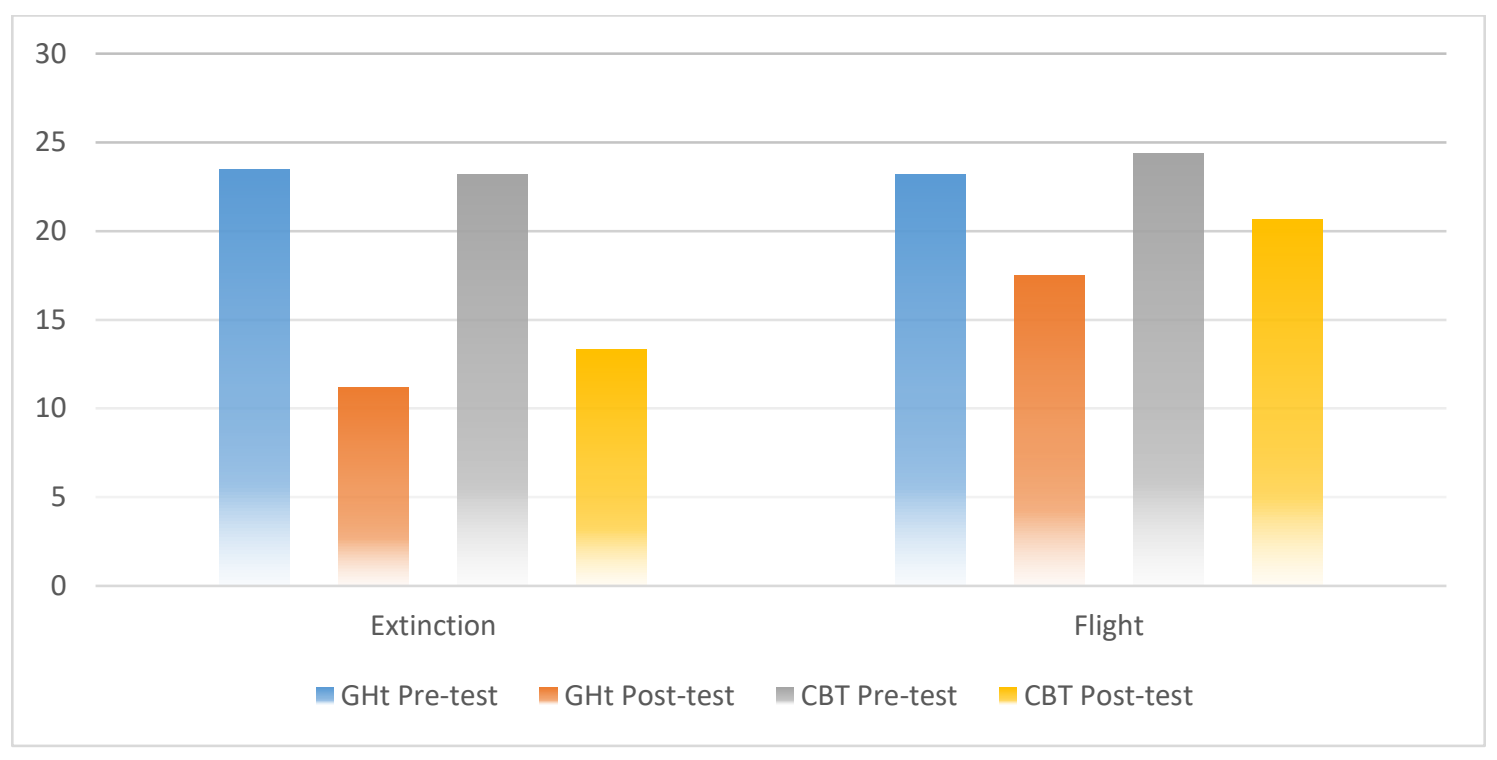

Figure 1. Comparison of CBT versus GHt results regarding Extinction and Flight components.

The researchers compared the pre and post SPIN and GPWQ components results of 15 participants who received CBGT with the results of 15 participants who received GHt. ANCOVA was used for the analysis of the results of the foregoing tests. In some components, the means were decreased from pre-test to post-test. Therefore, for the participants who received the therapeutic Hypnosis protocol for Extinction $(\mathrm{F}=6.963$, $P<0.05)$ and Flight $(\mathrm{F}=5.957, P<0.05)$, the results showed a significant difference from pre-intervention to post-intervention. Hence, the mean differences (for Extinction and Flight constituents) are not accidental. This demonstrates that the scores for the anxiety symptoms were higher in the pre-intervention phase. No significant difference was observed regarding the level of anxiety (SPIN) and brain/behavioral systems components such as Approach, Active Avoidance, Passive Avoidance, Fight, BAS, BIS, FFFS (check post-application values in Table 1).

\section{Discussion}

Previous studies have indicated that both CognitiveBehavioral Therapy and exposure can play a prominent role in habituation in the BIS and FFFS among brain behavioral systems, which consequently can leads to decrease in social anxiety (13,24-26). The exposure was a key component in both of the interventions applied -as forms of in-vivo exposure in CBT and invitro exposure in hypnosis- in this study. Analyzing the results, improvement in reduction of the anxious symptomatology was observed in both experimental groups. Also there were noticeable changes in brain/behavioral systems of both groups, which in some cases the hypnosis was more effective.

\section{Conclusion}

The aim of this study was to compare the short-term interventions in the treatment of social phobia and to observe the changes in brain/behavioral aspects of the disorder due to the differences between therapy approaches. Since hypnosis led to more significant changes in Extinction (BIS subsystem) and Flight (FFFS subsystem) as compared to the cognitive-behavioral therapy- which are two major components of the brain/behavioral systems involved in SAD-, it is recommended to consider hypnotherapy in treating general social phobia.

The results were found immediately after the completion of interventions and consisted of no follow-up. More comprehensive measures of BIS and FFFS would be useful for the field moving forward.

\section{Acknowledgment}

We thank Zanjan University of Medical Sciences for providing financial resources for this project. We thank Dr. Morris, Dr. Mensink, and Dr. Sherry (Dalhousie University) for their help in conducting CBGT protocol. We thank the Iranian Scientific Society of Clinical Hypnosis for their help in conducting GHt protocol and their supervision during the courses of treatments.

\section{Conflict of Interest}

The authors declared no conflicts of interest. 


\section{References}

1. Deborah C. Beidel BCF, Hersen M. Adult Psychopathology and Diangnosis. 7th ed. New Jersey: Wiley; 2014.

2. Association AP. Diagnostic and Statistical Manual of Mental Disorders DSM-5. 5th ed. Tehran: Arjmand Press; 2013.

3. Furmark T. Social phobia: Overview of community surveys. Acta Psychiatrica Scandinavica. 2002; 105: 84-93. [DOI:10.1034/j.1600-0447.2002.1r103.x] [PMID]

4. Kimbrel NA. A model of the development and maintenance of generalized social phobia. Clin Psychol Rev. 2008; 28(4): 529-612. [DOI:10.1016/j.cpr.2007.08.003] [PMID]

5. Ollendick TH, Hirshfeld-Becker DR. The developmental psychopathology of social anxiety disorder. Biologic \begin{tabular}{l} 
Psychiat. 2002; 51: 44-58. [DOI:10.1016/S0006- \\
\hline
\end{tabular} 3223(01)01305-1]

6. Rapee RM, Spence SH. The etiology of social phobia: Empirical evidence and an initial model. Clin Psychol Rev. 2004; 24: 737-67. [DOI:10.1016/j.cpr.2004.06.004] [PMID]

7. Kagan J, Reznick JS, Snidman N. The physiology and psychology of behavioral inhibition in children. Child Develop. 1987; 58: 1459-73. [DOI:10.2307/1130685]

8. Kimbrel NA. A model of development and maintenance of generalized social phobia. Clin Psychol Rev. 2008; 28(4): 592-612. [DOI:10.1016/i.cpr.2007.08.003] [PMID]

9. Corina L, Gomez R. Unique associations of reinforcement sensitivity theory dimensions with social interaction anxiety and social observation anxiety. Personal Individual Difference. $\quad 2014 ; \quad 60$ : 20-4. [DOI:10.1016/j.paid.2013.10.003]

10. Corr PJ. Reinforcement sensitivity theory and personality. Neuro Sci Biobehav Rev. 2004; 28: 317-32. [DOI:10.1016/j.neubiorev.2004.01.005] [PMID]

11. Gray JA. Neural systems, emotion, and personality. Madden JIE, editor. New York: Raven Press; 1991: 1991.

12. McNaughton N, Corr PJ. A two-dimensional neuropsychology of defense: Fear/anxiety and defensive distance. Neurosc Biobehav Rev. 2004; 28: 285-305. [DOI:10.1016/j.neubiorev.2004.03.005] [PMID]

13. Gray JA, McNaughton N. The neuropsychology of anxiety: An enquiry into the functions of the septo-hippocampal system. 2nd ed. New York Oxford University Press; 2000: 2000.

14. Coplan RJ, Wilson J, Frohlick SL, Zelenski J. A personoriented analysis of behavioral inhibition and behavioral activation in children. Pers Individ Dif. 2006; 41: 917-27. [DOI:10.1016/j.paid.2006.02.019]
15. Kashdan TB, Steger MF. Expanding the topography of social anxiety: Anvexperience-sampling assessment of positive emotions, positive events, and emotionvsuppression. Psychol Sci. 2006; 17: 120-8. [DOI:10.1111/j.1467-9280.2006.01674.x] [PMID]

16. Tillfors M, Furmark T, Marteinsdottir I, et al. Cerebral blood flow in social phobics during stressful speaking tasks: A PET-study. Am J Psychiat. 2001; 158: 1220-6. [DOI:10.1176/appi.ajp.158.8.1220] [PMID]

17. Tillfors M, Furmark T, Marteinsdottir I, Fredrikson M. Cerebral blood flow during anticipation of public speaking in social phobia: A PET-study. Biol Psychiat. 2002; 52: 1113-9. [DOI:10.1016/S0006-3223(02)01396-3]

18. Kimbrel NA, Meyer EC, DeBeer BB, et al. Reinforcement sensitivity and social anxiety in combat veterans. Pers Individ Dif. 2016; 98: 171-5. [DOI:10.1016/j.paid.2016.04.008] [PMID] [PMCID]

19. Olatunji BO, Cisler JM, Deacon BJ. Efficacy of cognitive behavioral therapy for anxiety disorders: A review of metaanalytic findings. Psychiat Clin North Am. 2010; 33: 55777. [DOI:10.1016/j.psc.2010.04.002] [PMID]

20. Holdevici I, Craciun B. Hypnosis in the treatment of patients with anxiety disorders. Soc Behav Sci. 2013; 78: 471-5. [DOI:10.1016/j.sbspro.2013.04.333]

21. Lynn SJ, Kirsch I. Essentials of clinical hypnosis: An Evidence-based Approach. American Psychological Association. 2006. 271 p. [DOI:10.1037/11365-000]

22. Halsband U WTG. Functional changes in brain activity after hypnosis in patients with dental phobia. J PhysiolParis. 2015; 109(4-6): 131-42. [DOI:10.1016/j.jphysparis.2016.10.001] [PMID]

23. Azad Fallah P, Dadsetan P, Ezhee J, Moazeni M. Stress: Work systems and brain/behaivioural changes IVIG a secretory. J Psychol. 1999; 3(9): 3-22.

24. Gould RA, Buckminster S, Pollack MH, Otto MW, Yap L. Cognitive-behavioral and pharmacological treatment for social phobia: A meta-analysis. Clin Psychol: Sci Pract. 1997; 4(4): 291-306. [DOI:10.1111/j.14682850.1997.tb00123.x]

25. Otto MW, Safren SA, Hearon BA. Mechanisms of action in the treatment of anxiety disorder. In socialanxiety: Clinical, Developmental and Social Perspectives. 3rd ed2014. [DOI:10.1016/B978-0-12-394427-6.00027-3]

26. Mattick RP, Peters L, Clark DM. Exposure and cognitive restructuring for social phobia: A controlled study. Behav Ther. 1989; 20(1): 3-23. [DOI:10.1016/S0005$\underline{\text { 7894(89)80115-7] }}$

\section{How to Cite This Article:}

